\title{
What is the Impact of Positive Airway Pressure in Nasal Polyposis? An Experimental Study
}

\author{
Leonardo Balsalobre $^{1}$ Rogerio Pezato ${ }^{1}$ [0 Joao Mangussi-Gomes ${ }^{1}$ Luciano Gregorio ${ }^{1}$ \\ Fernanda Louise Martinho Haddad ${ }^{1}$ Luiz Carlos Gregorio ${ }^{1}$ Reginaldo Fujita ${ }^{1}$
}

\author{
${ }^{1}$ ENT Research Laboratory, Department of Otolaryngology and Head \\ and Neck Surgery, Universidade Federal de São Paulo, SP, Brazil \\ Int Arch Otorhinolaryngol 2019;23:147-151.
}

Address for correspondence Rogerio Pezato, MD, PhD,

Departamento de Otolaringologia e Cirurgia da Cabeça e Pescoço, Universidade Federal de São Paulo, Rua Pedro de Toledo 847, Sao Paulo, SP, 04025011, Brazil (e-mail: pezatobau@gmail.com).

\begin{abstract}
Keywords

- chronic rhinosinusitis

- endoscopy

- extracellular matrix

- nasal airflow dynamics

- rhinosinusitis

Introduction It has been hypothesized that increasing the interstitial hydrostatic pressure within the sinonasal mucosa of patients with nasal polyposis (NP) might decrease the size of nasal polyps.

Objective To evaluate the effects of positive airway pressure, delivered by a continuous positive airway pressure (CPAP) device, in patients with NP and in control subjects.

Methods Twelve patients with NP and 27 healthy subjects were exposed to CPAP $(20 \mathrm{~cm} \mathrm{H2O})$ for 2 hours. Visual analog scale (VAS), Nasal Obstruction Symptom Evaluation (NOSE) scale, acoustic rhinometry (AR), peak nasal inspiratory flow (PNIF) and nasal endoscopy (NE-Meltzer polyp grading system) were performed before and after the intervention, for all patients.

Results The control group showed a significant worsening in nasal obstruction symptoms, as measured by VAS and NOSE $(p<0.01)$, and a significant decrease in nasal patency, as measured by the PNIF and AR $(p<0.01)$. For the NP group, VAS, NOSE, and AR did not differ significantly $(p=0.72, p=0.73$, and $p=0.17$, respectively), but PNIF values worsened $(p=0.04)$ after exposure to CPAP. There was a statistically significant reduction in the nasal polyps' size $(p=0.04)$.

Conclusions Positive pressure worsened the nasal obstruction symptoms and decreased objective parameters of nasal patency in control subjects. In patients with NP, exposure to CPAP reduced the nasal polyps' size, and the nasal patency, as measured by PNIF. However, it had no significant effects in AR and in nasal obstruction symptoms.
\end{abstract}

\section{Introduction}

Recent studies have shown that nasal polyposis (NP) is characterized not only by chronic inflammation of the sinonasal mucosa, but also by a biomechanical disequilibrium between oncotic and hydrostatic pressures within the vessels and interstitial space. In normal subjects, and in the context of an inflammatory process, the equilibrium

(D) Rogerio Pezato's ORCID is https://orcid.org/0000-0002-98134466.

received

April 19, 2018

accepted

October 6, 2018

published online

March 7, 2019 10.1055/s-0038-1676095. ISSN $1809-9777$. between these factors is responsible for limiting to a certain extent the extravasation of fluid to the interstitial space. In NP, however, there is a partial dysfunction of these equilibrium mechanisms, which facilitates the development of edema and alters the tissue remodeling process ( - Fig. 1). ${ }^{1-3}$

In this sense, we have hypothesized that increasing the interstitial hydrostatic pressure might counteract the increased oncotic pressure and further mitigate the development of NP. Besides decreasing the size of nasal polyps, the increased interstitial hydrostatic pressure could also improve the nasal patency and reduce symptoms of nasal
Copyright @ 2019 by Thieme Revinter Publicações Ltda, Rio de Janeiro, Brazil
License terms

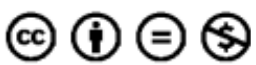




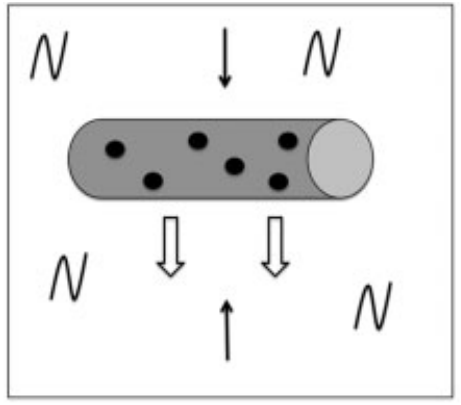

A) Healthy tissue $\mathrm{OP}=\mathrm{HP}$

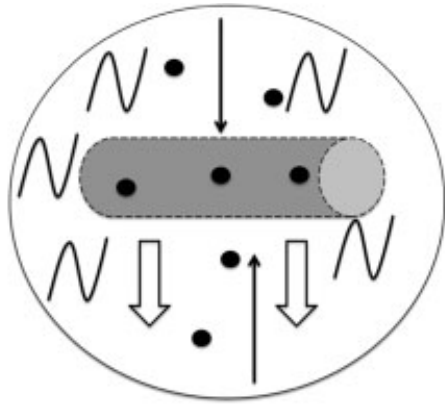

B) Inflamed tissue $\uparrow O P / \uparrow H P$

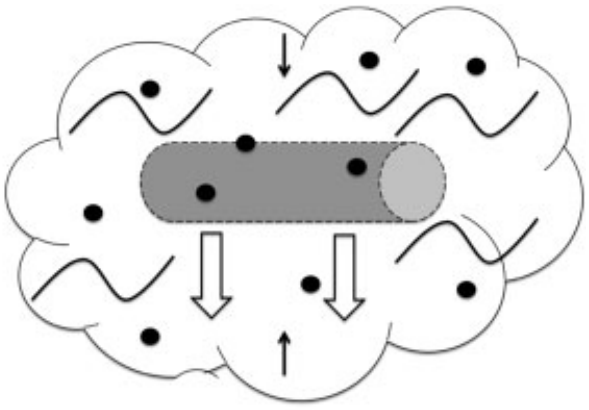

C) Nasal polyp tissue $\uparrow O P / \downarrow_{H P}$

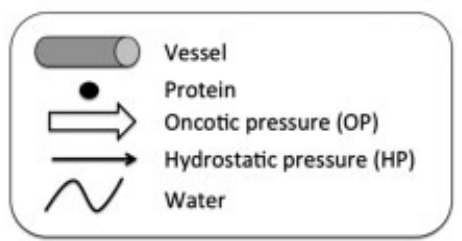

Fig. 1 Illustration showing an increase in capillary permeability due to inflammation, with protein loss and increasing tissue oncotic pressure (B and C), and, consequently, extravasation of water to the interstitial space and edema. (B) compensatory increase in interstitial hydrostatic pressure in response to increasing oncotic pressure, thus limiting edema. (C) less marked increase in interstitial hydrostatic pressure in response to increasing oncotic pressure, facilitating the greater loss of water from the capillary lumen into the tissues and, consequently, increased edema.

obstruction in patients with NP. ${ }^{4}$ A rational way to test this theory is to deliver continuous positive airway pressure (CPAP) to the nasal cavities of NP patients.

The objective of this study was to evaluate the effects of positive pressure, delivered by a continuous positive airway pressure (CPAP) device, on patients with NP and in control subjects, with special interest in nasal obstruction symptoms, objective nasal patency parameters, and nasal polyps' size.

\section{Methods}

\section{Design, Setting, and Study Population}

An analytic, observational, and cross-sectional study was conducted at a tertiary otorhinolaryngology referral center, from January 2016 to August 2016. Participants were divided in two groups. The NP group included only those individuals with a recent diagnosis of NP, according to the European Position Paper on Rhinosinusitis and Nasal Polyps 2012 (EPOS 2012) guidelines, ${ }^{5}$ and with polyps graded $\geq 1$ according to the Meltzer polyp grading system. ${ }^{6}$ The control group included only healthy individuals. For both groups, participants should be older than 18 years-old and younger than 65 years-old, and the following were excluded:

- Those using or who had recently used ( $<4$ weeks) antihistaminic or antihypertensive drugs, topical vasoconstrictors, systemic vasodilators, or systemic/topic corticosteroids;

- Those with severe septal deviation precluding nasal endoscopy or the use of nasal cannulas for acoustic rhinometry;

- Those with a present or past history of tumors, prior sinonasal surgery, known diagnosis of obstructive sleep apnea, and/or use of CPAP;
- Those with a present or past history of smoking or illicit drug use.

For the control group, those who had a present or recent past ( $<4$ weeks) history of sinonasal infections and/or inflammation were also excluded.

After application of inclusion and exclusion criteria, 13 and 27 subjects were included in the NP and control groups, respectively. Of these, 12 and 27 subjects were considered for the final analysis, respectively, because 1 patient from the NP group did not tolerate the intervention (CPAP). In the NP group, 5 (41.6\%) participants were women and 7 (58.4\%) were men, with ages ranging from 34 to 65 years. Of the 27 individuals in the control group, 10 (37.0\%) were women and $17(63.0 \%)$ were men, with ages ranging from 18 to 43 years.

This study conforms to recognized ethical standards and to the Declaration of Helsinki, and it was approved by the local institutional review board (n. 897.279, 12/2015). Written informed consent was obtained from every participant included in the study.

\section{Exposure to Positive Pressure-CPAP}

All subjects were exposed to CPAP, delivered by a mechanical device (F\&P Icon, Fisher \& Paykel Healthcare Ltd., Auckland, New Zealand) attached to a nasal mask (Meridian Nasal Mask, ResMed Ltd., Bella Vista, Australia) for 2 hours, at a pressure of $20 \mathrm{~cm} \mathrm{H}_{2} \mathrm{O}$. All patients were in a comfortable sitting position during the whole procedure, and air leak through the mask was ruled out for all cases. No topical medications were used before or after the intervention.

\section{Analyzed Variables}

The following parameters were measured for all participants, immediately before and after exposure to the CPAP: 
- Visual analog scale (VAS) for nasal obstruction symptoms;

- Nasal Obstruction Symptom Evaluation (NOSE) scale;

- Nasal endoscopy (NE);

- Acoustic rhinometry (AR);

- Peak nasal inspiratory flow (PNIF).

\section{Visual Analog Scale (VAS) and Nasal Obstruction} Symptom Evaluation (NOSE) Scale

For the evaluation of nasal obstruction symptoms, all participants were asked to score their nasal obstruction severity in a $10-\mathrm{cm}$ VAS, ranging from " 0 " to " 10 ," with "0" meaning "complete absence of nasal obstruction," and "10" meaning "complete nasal obstruction."

The NOSE scale, previously adapted to Brazilian Portuguese, ${ }^{7}$ was also administered and calculated for all participants, with scores ranging from 0 to 12 . Because of the characteristics of this study, the assessment of nasal obstruction during sleep and exercise was not considered.

\section{Nasal Endoscopy (NE)}

Nasal endoscopy was performed in all patients. An 18-cm, 4$\mathrm{mm}$, 0-degree rigid endoscope (Hopkins II, Karl Storz Ltd., Tuttlingen, Germany) attached to a video camera system (IKM51H / IK-CU51 Imaging System, Toshiba America Inc., Irvine-CA, USA), monitor (OEV 141, Olympus Optical Ltd., Barlett-TN, USA), and an Innova Light \& Image FX 300R light source (Innova Technik, Cajamar, SP, Brazil) were used. Images were digitally recorded with an HD PVR Rocket video capture device (Hauppauge Inc., Hauppauge, NY, USA).

Two blinded evaluators, both of whom were experienced rhinologists, watched every NE recorded, and, together, classified the severity of NP for every nasal cavity, according to the Meltzer polyp grading system ${ }^{6}$ ( $\boldsymbol{\sim}$ Table $\mathbf{1}$ ).

\section{Acoustic Rhinometry and PNIF}

For the objective evaluation of nasal patency, all patients underwent AR and PNIF, before and after exposition to CPAP.

The AR was performed without administration of vasoconstrictors, with a calibrated acoustic rhinometer and the A1 Acoustic Rhinometer software (GM Instruments Ltd., Kilwinning, UK). The test was conducted as standardized by the International Standardization Committee on Objective Assessment of the Nasal Airway. ${ }^{8}$ Each participant remained for 30 minutes in an air-conditioned room (temperature set to $21^{\circ} \mathrm{C}$ before measurement, and ambient humidity kept in the 50-60\% range); the head of each participant was stabilized to ensure proper positioning of the pulse tube; petroleum jelly

Table 1 Meltzer polyp grading system

\begin{tabular}{|l|l|}
\hline Endoscopic appearance & Score \\
\hline No visible nasal polyps & 0 \\
\hline $\begin{array}{l}\text { Small amount of polypoid disease confined } \\
\text { within the middle meatus }\end{array}$ & 1 \\
\hline Multiple polyps occupying the middle meatus & 2 \\
\hline Polyps extending beyond the middle meatus & 3 \\
\hline Polyps completely obstructing the nasal cavity & 4 \\
\hline
\end{tabular}

was used to prevent air leak; all participants were instructed to control their breathing.

At least three curves were obtained for each nostril-after each measurement, the nosepiece was removed, reconnected, and a new measurement was then obtained; the results were considered adequate if the coefficient of variability was lower than $10 \%$; the recorded curves were used to obtain a mean curve for each nostril; the values of these mean curves were then analyzed. All examinations were performed by the same investigator, experienced in AR. The cross-sectional area between the distances of 0 and $5 \mathrm{~cm}$, expressed in $\mathrm{cm}^{2}$, was used for objective comparison of findings.

The measurement of PNIF was performed with an InCheck Nasal Inspiratory Flow Meter portable device (Clement Clarke International Ltd., Essex, UK) equipped with an aircushioned facemask. The PNIF was measured with the participant in the standing position, at three consecutive times with a 1-minute interval between measurements. The results were obtained immediately, and the average of the measures was considered for the final analysis.

\section{Statistical Analysis}

Data was plotted and analyzed in the Statistical Package for the Social Sciences (SPSS) v. 22 (IBM Corp., Armonk, NY, USA) and Prism $v .7$ (GraphPad Software Inc., La Jolla, CA, USA) software environments. The Wilcoxon, Mann-Whitney $U$, and Fisher exact tests were used to assess differences within and between groups. The binomial sign test was used for estimation of statistical significance for the Meltzer score before and after exposure to CPAP for the NP group. In all cases, $p$ values $<0.05$ were considered statistically significant.

\section{Results}

There was no statistically significant difference in gender distribution between groups $(p=1.00)$. The participants in the control group were significantly younger than patients in the NP group $(p<0.01)(-$ Table 2$)$.

\section{Effects of Positive Pressure on Symptoms of Nasal Obstruction (VAS and NOSE)}

For the control group, there was a significant deterioration in nasal obstructive symptoms, as measured by the VAS and NOSE, after exposure to CPAP $(p<0.01)$. In the NP group, VAS and NOSE did not differ significantly after exposure to CPAP ( $p=0.72$ and $p=0.73$, respectively).

Table 2 Demographic characteristics for the control group and the NP group

\begin{tabular}{|l|l|l|l|l|}
\hline & & $\begin{array}{l}\text { Control } \\
\text { Group }\end{array}$ & NP Group & $p$ \\
\hline \multirow{2}{*}{ Gender } & Female (\%) & $10(37.0 \%)$ & $5(41.6 \%)$ & 1.00 \\
\cline { 2 - 4 } & Male (\%) & $17(63.0 \%)$ & $7(58.4 \%)$ & \\
\hline Age & $\begin{array}{l}\text { Age } \\
\text { average } \\
( \pm \text { SD })\end{array}$ & $24.03 \pm 4.17$ & $48.17 \pm 10.94$ & $<0.01$ \\
\hline
\end{tabular}

Abbreviations: NP, nasal polyposis; SD, standard deviation. 


\section{Effects of Positive Pressure on Nasal Patency (AR and PNIF)}

Anterior nasal cavity volume and PNIF decreased significantly in the control group after CPAP use $(p<0.01)$. For the NP group, the values of PNIF worsened $(p=0.04)$, but there was no significant alteration in the AR after the intervention $(p=0.17)$.

\section{Effects of Positive Pressure on NE}

There was a statistically significant reduction in nasal polyps' size for the NP group, as measured by NE and the Meltzer

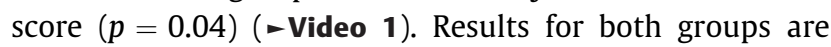
summarized in -Table 3.

\section{Video 1}

Nasal endoscopic evaluation of the right nasal cavity of a patient with nasal polyposis that underwent exposition to continuous positive airway pressure (CPAP) for 2 hours. Note the reduction in the polyps' size after intervention. Online content including video sequences viewable at:

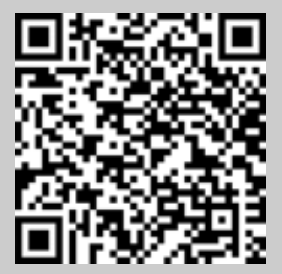

www.thieme-connect.com/products/ejournals/html/10.1055/s-00381676095 .

\section{Discussion}

\section{Synopsis of Key Findings}

Positive pressure significantly worsened the nasal obstruction symptoms and decreased the objective parameters of nasal patency in control subjects. In patients with NP, exposure to CPAP reduced the nasal polyps' size and the nasal patency, as measured by PNIF. However, it had no significant effects on AR and on nasal obstruction symptoms.

\section{Strengths and Limitations of this Study}

This study presents some limitations, with its small sample size being, arguably, the most important one. For instance, increasing the number of participants could yield statistically significant results for nasal patency parameters in the NP group. It could be also questioned whether the exposure to CPAP for longer periods of time, set at different pressures, or with the patients in different positions, could yield different results. Indeed, patients with obstructive sleep apnea syndrome (OSA) use CPAP for 8 sleeping-hours and in the horizontal position. These factors affect the lymphatic and venous drainage of the nasal mucosa and could also have influenced the results of this study. ${ }^{9}$

Moreover, the long-term effects of CPAP on NP were not evaluated. Considering that oncotic pressure does not change with time, but hydrostatic pressure decreases after CPAP exposure is ended, the polyps could have returned to their preexposure size after a few hours/days. A secondary evaluation would be ideal to assess what are the real permanent effects of CPAP on the interstitial hydrostatic pressure and the polyps' volume.

Nonetheless, this study has the great advantage of experimentally determining the effects of positive pressure on NP, which, to the best of our knowledge, had never been done before. It was also possible to compare these effects in healthy individuals. The results presented are worthy to be taken into consideration in the understanding of the NP pathophysiology.

\section{Interpretation of Findings and Comparison with Other Studies}

Previous studies have disclosed differences in the extracellular matrix composition and the remodeling process that takes place in the sinonasal mucosa of patients with NP, mainly due to differences in transforming growth factor beta (TGF- $\beta$ ) and metalloproteinases expression. ${ }^{10,11}$ This has raised the suspicion for a biomechanical disequilibrium in the pathogenesis of $\mathrm{NP},{ }^{11-14}$ which would facilitate mucosal growth excess in the presence of chronic inflammation. ${ }^{3}$ Subsequent studies showed that immune regulatory cells, such as dendritic cells and mesenchymal stem cells, could also be involved in the maintenance of chronic inflammation, abnormal remodeling, and biomechanical imbalance, typically found in patients with NP. ${ }^{15-18}$

Table 3 Effects of continuous positive airway pressure in patients with nasal polyposis and in control subjects

\begin{tabular}{|l|l|l|l|l|l|l|l|}
\hline \multicolumn{2}{|l|}{ Characteristics (mean \pm SD) } & \multicolumn{2}{l|}{ Control Group } & \multicolumn{2}{l|}{ NP Group } \\
\cline { 3 - 8 } \multicolumn{2}{l|}{} & Pre-CPAP & Post-CPAP & $p^{*}$ & Pre-CPAP & Post-CPAP & $p^{*}$ \\
\hline \multirow{2}{*}{$\begin{array}{l}\text { Nasal obstruction } \\
\text { symptoms }\end{array}$} & VAS & $2.66 \pm 1.8$ & $4.55 \pm 2.6$ & $<0.01$ & $4.75 \pm 2.43$ & $5.25 \pm 3.06$ & 0.72 \\
\cline { 2 - 8 } & NOSE & $2.74 \pm 1.85$ & $5.00 \pm 3.00$ & $<0.01$ & $6.62 \pm 2.97$ & $6.13 \pm 2.59$ & 0.73 \\
\hline \multirow{2}{*}{ Nasal patency } & AR & $11.98 \pm 3.6$ & $10.08 \pm 2.4$ & $<0.01$ & $5.06 \pm 2.83$ & $4.36 \pm 1.78$ & 0.17 \\
\cline { 2 - 8 } & PNIF & $127.15 \pm 50.2$ & $112.37 \pm 54.8$ & $<0.01$ & $117.5 \pm 53.92$ & $92.5 \pm 43.99$ & 0.04 \\
\hline $\begin{array}{l}\text { Nasal endoscopic } \\
\text { evaluation }\end{array}$ & Meltzer Score & - & - & - & $6.08 \pm 1.68 \dagger$ & $5.42 \pm 1.88 \dagger$ & $0.04 \ddagger$ \\
\hline
\end{tabular}

Abbreviations: CPAP, continuous positive airway pressure; NOSE, nasal obstruction symptoms evaluation; NP, nasal polyposis; PNIF, peak nasal inspiratory flow; SD, standard deviation; VAS, visual analog scale.

${ }^{*} p$-values for comparisons within-group; $†$ Meltzer score for both nasal cavities. $\ddagger$ Binomial sign test. 
Recently, these biomechanical differences were experimentally demonstrated in the sinonasal mucosa of patients with NP. ${ }^{1-3}$ The biomechanical dysfunction found in NP is characterized by a deficiency in the ability to properly raise interstitial hydrostatic pressure in response to fluid extravasation during the inflammatory process, a mechanism that is crucial to limit the development of edema, and is closely related to the extracellular matrix composition. ${ }^{1-3}$

In this context, it has been shown that synechial tissues exhibit biomechanical properties similar to those of the healthy nasal mucosa. Thus, fibrosis could be a possible remodeling mechanism that would enhance the interstitial hydrostatic pressure in NP. ${ }^{4}$ In the present study, the interstitial hydrostatic pressure in NP was indirectly increased through the acute and transient delivery of CPAP to the nasal cavity. A significant reduction in the nasal polyps' size in patients with NP was observed after exposure to CPAP. This suggests that, in fact, increasing interstitial hydrostatic pressure in nasal polyps, even if indirectly, acutely and transiently, can possibly affect the pathophysiology of NP.

In patients with OSA, the use of CPAP (especially at high titers, such as $20 \mathrm{~cm}^{2}$ ), causes nasal obstruction and local irritation symptoms, ultimately leading to treatment intolerance and nonadherence. In this study, the control group showed marked nasal obstruction worsening both in subjective (VAS, NOSE) and objective measurements (AR and PNIF), which is in line with previous studies. ${ }^{19}$

In the NP group, although PNIF values worsened after exposure to CPAP, there was no significant worsening of nasal obstruction symptoms or in AR measurements. Continuous positive airway pressure also determined a decrease in the nasal polyps' size in the NP group. The reason for these observations is still unclear and admits at least two interpretations:

1) The reduction in the nasal polyps' size prevented significant worsening of nasal patency parameters and nasal obstruction symptoms in patients with NP, in spite of the worsening PNIF;

2) Or, although CPAP determined a decrease in the nasal polyps' size, no improvement in nasal obstruction symptoms and nasal patency parameters were observed. This could be explained by the fact that patients with NP already have significantly decreased nasal patency and obstructive nasal symptoms at baseline, and this would prevent further deterioration in obstructive parameters after exposure to CPAP.

\section{Clinical Applicability}

Although it was not the primary objective of this study, we conclude that CPAP could be used as a therapeutic option, especially in patients with OSA and NP, prior or not to endoscopic endonasal surgery. Future studies could address such possibilities.

\section{Conclusion}

Positive pressure significantly worsened the nasal obstruction symptoms and decreased the objective parameters of nasal patency in control subjects. In patients with NP, exposure to CPAP reduced the nasal polyps' size, and the nasal patency, as measured by PNIF. However, it had no significant effects on AR and on nasal obstruction symptoms.

\section{References}

1 Pezato R, Voegels RL, Pinto Bezerra TF, Perez-Novo C, Stamm AC, Gregorio LC. Mechanical disfunction in the mucosal oedema formation of patients with nasal polyps. Rhinology 2014;52(02):162-166

2 Pezato R, Voegels RL, Stamm AC, Gregório LC. Why we should avoid using inferior turbinate tissue as control to Nasal Polyposis studies. Acta Otolaryngol 2016;136(09):973-975

3 Pezato R, Voegels RL. Why do we not find polyps in the lungs? Bronchial mucosa as a model in the treatment of polyposis. Med Hypotheses 2012;78(04):468-470

4 Gregório L, Pezato R, Felici RS, Kosugi EM. Fibrotic Tissue and Middle Turbinate Exhibit Similar Mechanical Properties. Is Fibrosis a Solution in Nasal Polyposis? Int Arch Otorhinolaryngol 2017; 21(02):122-125

5 Fokkens WJ, Lund VJ, Mullol J, et al. EPOS 2012: European position paper on rhinosinusitis and nasal polyps 2012. A summary for otorhinolaryngologists. Rhinology 2012;50(01):1-12

6 Meltzer EO, Hamilos DL, Hadley JA, et al; Rhinosinusitis Initiative. Rhinosinusitis: developing guidance for clinical trials. J Allergy Clin Immunol 2006;118(5, Suppl):S17-S61

7 Bezerra TFP, Padua FGM, Pilan RRM, Stewart MG, Voegels RL. Cross-cultural adaptation and validation of a quality of life questionnaire: the Nasal Obstruction Symptom Evaluation questionnaire. Rhinology 2011;49(02):227-231

8 Clement P a. R, Gordts F, Standardisation Committee on Objective Assessment of the Nasal Airway, IRS, and ERS. Consensus report on acoustic rhinometry and rhinomanometry. Rhinology 2005 Sep; 43 (03):169-179

9 Suman JD. Current understanding of nasal morphology and physiology as a drug delivery target. Drug Deliv Transl Res 2013;3(01):4-15

10 Liu Z, Gao Q, Zhang S, You X, Cui Y. Expression of tenascin and fibronectin in nasal polyps. J Huazhong Univ Sci Technolog Med Sci 2002;22(04):371-374

11 Van Bruaene N, Derycke L, Perez-Novo CA, et al. TGF-beta signaling and collagen deposition in chronic rhinosinusitis. J Allergy Clin Immunol 2009;124(02):253-259, 259.e1-259.e2

12 Li X, Meng J, Qiao X, et al. Expression of TGF, matrix metalloproteinases, and tissue inhibitors in Chinese chronic rhinosinusitis. J Allergy Clin Immunol 2010;125(05):1061-1068

13 Balsalobre L, Pezato R, Perez-Novo C, et al. Epithelium and stroma from nasal polyp mucosa exhibits inverse expression of TGF- $\beta 1$ as compared with healthy nasal mucosa. J Otolaryngol Head Neck Surg 2013;42:29

14 Figueiredo CR, Santos RP, Silva IDCG, Weckx LLM. Microarray cDNA to identify inflammatory genes in nasal polyposis. Am J Rhinol 2007;21(02):231-235

15 Pezato R, de Almeida DC, Bezerra TF, et al. Immunoregulatory effects of bone marrow-derived mesenchymal stem cells in the nasal polyp microenvironment. Mediators Inflamm 2014;2014:583409

16 Pezato R, Pérez-Novo CA, Holtappels G, et al. The expression of dendritic cell subsets in severe chronic rhinosinusitis with nasal polyps is altered. Immunobiology 2014;219(09):729-736

17 Perez-Novo C, Pezato R. Dendritic cell subset expression in severe chronic rhinosinusitis with nasal polyps. Curr Opin Allergy Clin Immunol 2017;17(01):1-4

18 de Oliveira PWB, Pezato R, Agudelo JSH, et al. Nasal Polyp-Derived Mesenchymal Stromal Cells Exhibit Lack of Immune-Associated Molecules and High Levels of Stem/Progenitor Cells Markers. Front Immunol 2017;8:39

19 Zozula R, Rosen R. Compliance with continuous positive airway pressure therapy: assessing and improving treatment outcomes. Curr Opin Pulm Med 2001;7(06):391-398 